\title{
Diffusion Tensor Imaging Correlates of Cognitive-Motor Decline in Normal Aging and Increased Alzheimer's Disease Risk
}

\author{
Kara M. Hawkins ${ }^{\mathrm{a}, \mathrm{b}, \mathrm{c}}$, Aman I. Goyal ${ }^{\mathrm{d}}$ and Lauren E. Sergio ${ }^{\mathrm{a}, \mathrm{b}, \mathrm{c}, *}$

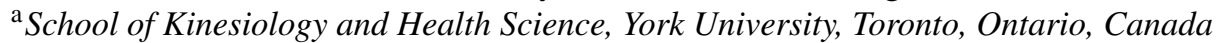 \\ ${ }^{\mathrm{b}}$ Centre for Vision Research, York University, Toronto, Ontario, Canada \\ ${ }^{\mathrm{c}}$ Neuroscience Graduate Diploma Program, York University, Toronto, Ontario, Canada \\ ${ }^{\mathrm{d}}$ Neuroimaging Laboratory, York University, Toronto, Ontario, Canada
}

Accepted 29 September 2014

\begin{abstract}
Alzheimer's disease (AD) is typically associated with impairments in memory and other aspects of cognition, while deficits in complex movements are commonly observed later in the course of the disease. Recent studies, however, have indicated that subtle deteriorations in visuomotor control under cognitively demanding conditions may in fact be an early identifying feature of AD. Our previous work has shown that the ability to perform visuomotor tasks that rely on visual-spatial and rulebased transformations is disrupted in prodromal and preclinical AD. Here, in a sample of 30 female participants (10 young: mean age $=26.6 \pm 2.7,10$ low $\mathrm{AD}$ risk: mean age $=58.7 \pm 5.6$, and 10 high $\mathrm{AD}$ risk: mean age $=58.5 \pm 6.9$ ), we test the hypothesis that these cognitive-motor impairments are associated with early AD-related brain alterations. Using diffusion-weighted magnetic resonance imaging, we examined changes in white matter (WM) integrity associated with normal aging and increased AD risk, and assessed the relationship between these underlying WM alterations and cognitive-motor performance. Our whole-brain analysis revealed significant age-related declines in WM integrity, which were more widespread in high relative to low AD risk participants. Furthermore, analysis of mean diffusivity measures within isolated WM clusters revealed a stepwise decline in WM integrity across young, low AD risk, and high AD risk groups. In support of our hypothesis, we also observed that lower WM integrity was associated with poorer cognitive-motor performance. These results are the first to demonstrate a relationship between AD-related WM alterations and impaired cognitive-motor control. The application of these findings may provide a novel clinical strategy for the early detection of individuals at increased AD risk.
\end{abstract}

Keywords: Aging, Alzheimer's disease, diffusion tensor imaging, geriatric assessment, magnetic resonance imaging, motor skills, movement, neurodegenerative disorder

\section{INTRODUCTION}

Over the past several years, many brain-imaging studies have investigated the neural underpinnings of Alzheimer's disease (AD). In 2009, a meta-analysis of these studies revealed that, aside from the commonly known structural atrophy in trans-entorhinal and hippocampal regions, hypometabolism and hypoperfu-

*Correspondence to: Lauren E. Sergio, PhD, York University, School of Kinesiology and Health Science, 4700 Keele Street, Toronto, Ontario, M3J 1P3, Canada. Tel.: +1 416736 2100/ext. 33641; Fax: +1 416736 5774; E-mail: 1sergio@yorku.ca. sion in the inferior parietal lobule and precuneus are also prevalent features of early AD [1]. Furthermore, recent neuroimaging studies employing diffusion tensor imaging (DTI) in mild cognitive impairment and $\mathrm{AD}$ have reported white matter (WM) compromise affecting several major fiber tracts forming parietalfrontal, interhemispheric, and hippocampal-cortical connections, including the inferior fronto-occipital fasciculus (IFOF), inferior longitudinal fasciculus (ILF), superior longitudinal fasciculus (SLF), corpus callosum (CC), and cingulum (CG) bundle [2-5]. Microstructural declines in the CG, ILF, and IFOF have also been found in cognitively normal women 
at increased risk of $\mathrm{AD}$ due to family history and apolipoprotein E4 (ApoE4) genotype, years before the expected onset of cognitive symptoms [6,7].

While AD dementia is typically associated with declines in cognition and short-term memory resulting from hippocampal atrophy [8], these clinical symptoms only present themselves after significant damage to the brain has already occurred [9]. Thus, neuroimaging studies including individuals in the preclinical (i.e., increased genetic risk) and prodromal (i.e., mild cognitive impairment) stages of $\mathrm{AD}$ are essential for the development of early detection strategies. Notably, the alterations in parietal areas and declines in WM integrity that have been observed in these preclinical/prodromal studies provide insight into other behaviors that may be affected even earlier in the disease process. Specifically, parietal cortex plays an important role in transforming visual-spatial information into goal-directed actions [10-13]. Further, WM tracts forming hippocampal-parietal and parietal-frontal connections are required to successfully transform spatial representations and contextual information into accurate motor outputs [14-16]. These observations suggest that early neurodegeneration may have an impact on rule-based motor control. Accordingly, impairments in visuomotor control under non-standard conditions, in which the correspondence between vision and action is not direct [17], have been observed in early and preclinical AD [18-26]. Given the potential impact of AD-related brain alteration on motor control and the close relationship between cognition and action [27], the aim of the present study is to examine the underlying structural connectivity associated with impaired cognitive-motor performance observed in individuals at increased AD risk. Using DTI and high-resolution magnetic resonance imaging (MRI), we test the hypothesis that kinematic measures from a cognitively demanding visuomotor task are associated with identifiable brain alterations similar to those observed in early AD, and thus may be useful in identifying individuals at increased AD risk.

\section{MATERIALS AND METHODS}

\section{Subjects}

Thirty right-handed female participants were included in this study: 10 healthy young adults (mean age $=26.6 \pm 2.7$ ), 10 low AD risk older adults (mean age $=58.7 \pm 5.6$ ), and 10 high $\mathrm{AD}$ risk older adults (mean age $=58.5 \pm 6.9$ ). Individuals classified as high $\mathrm{AD}$ risk scored at or above age- and education- adjusted norms on the Montreal Cognitive Assessment (MoCA) and reported either a maternal, multiple, or early-onset family history of AD. While earlyonset $\mathrm{AD}$ is known to be associated with the rare familial form of the disease [28], individuals with late-onset $\mathrm{AD}$ in their immediate family have also been shown to be at increased risk [29-31], especially if multiple family members are affected [32]. Paternal family history alone was not included in the high $\mathrm{AD}$ risk classification based on recent evidence that paternal history may not carry the same increased risk as maternal history [33-35]. Individuals classified as low AD risk were aged-matched with high AD risk participants, reported no dementia of any type within their known family history, scored at or above age- and education-adjusted norms on the MoCA, and expressed no memory complaints beyond normal expectations for their age. Exclusion criteria included vision or upper-limb impairments, any medical condition that would hinder task performance (e.g., severe arthritis), any neurological or psychiatric illnesses (e.g., schizophrenia, depression, alcoholism, epilepsy, Parkinson's disease), and any history of stroke or severe head injury. High and low $\mathrm{AD}$ risk participants also underwent ApoE genotyping, which supported the notion of increased genetic risk in our family history group (i.e., $80 \%$ of this sample were ApoE4 carriers, compared to only $20 \%$ of the no family history group). Demographic characteristics for all study participants are summarized in Table 1 . Our decision to focus on female participants in the current study was based on a few factors: 1) the prevalence of $\mathrm{AD}$ is higher in women, emphasizing the importance of studies specifically tailored toward early detection in this population [36,37],2) recent evidence suggesting that sex-differences in the efficacy of ApoE in redistributing myelin cholesterol during nerve repair may increase vulnerability to the degradation of WM tracts specifically in women who carry the ApoE4 allele [38], and 3) to avoid sex-related confounds inherent in brain imaging studies. The study protocol was approved by

Table 1

Demographic characteristics of subjects

\begin{tabular}{lccc}
\hline & Young & $\begin{array}{c}\text { Low AD } \\
\text { risk }\end{array}$ & $\begin{array}{c}\text { High AD } \\
\text { risk }\end{array}$ \\
\hline Number & 10 & 10 & 10 \\
Age (SD) & $26.6(2.7)$ & $58.7(5.6)$ & $58.5(6.9)$ \\
Years of education (SD) & - & $17.9(3.1)$ & $16.8(3.1)$ \\
MoCA score (SD) & - & $27.9(1.7)$ & $28.3(2.2)$ \\
ApoE genotype (\% E4 carriers) & - & $20 \%$ & $80 \%$ \\
\hline
\end{tabular}

SD, standard deviation; MoCA, Montreal cognitive assessment; ApoE, apolipoprotein E. 
the Human Participants Review Sub-Committee, York University's Ethics Review Board, and conformed to the standards of the Canadian Tri-Council Research Ethics guidelines.

\section{Visuomotor assessment}

A detailed description of our visuomotor assessment is provided in Hawkins and Sergio [24]. Briefly, in the current study, participants were tested on two visuomotor transformation tasks presented on an Acer Iconia 6120 dual-touchcreen tablet: One standard task in which the spatial location of the viewed target and the required movement were the same, and one cognitively demanding non-standard task (plane dissociated+feedback reversal) in which the location of the viewed target was dissociated from the required movement (Fig. 1A). Task conditions were presented in a randomized order consisting of five pseudo-randomly presented trials to each of four peripheral targets (from a common central 'home' target), for a total of 20 trials per condition and 40 trials per participant (Fig. 1B). Specifically, in the standard condition participants slid their finger directly to targets on the vertical tablet,

A
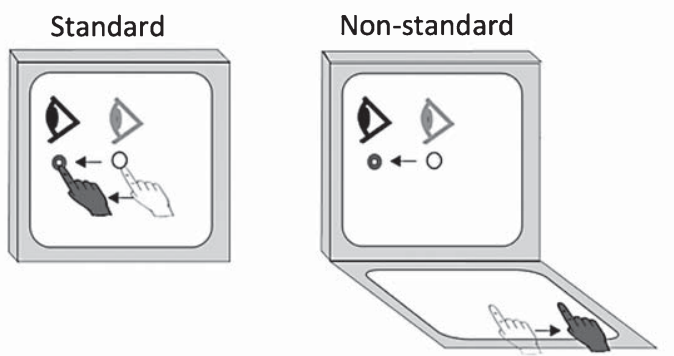

B

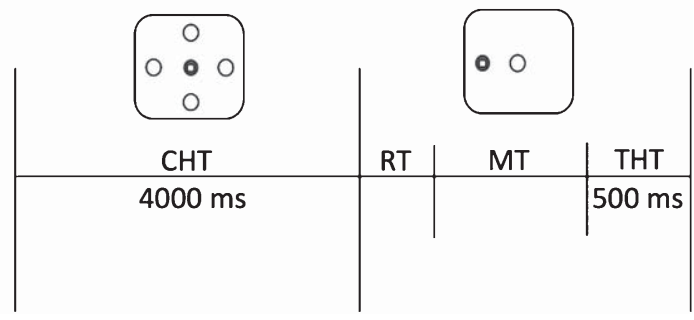

Fig. 1. A) Schematic drawing of the two experimental conditions. Light grey circle, eye, and hand symbols denote the starting position for each trial (i.e., the home target). Dark grey eye and hand symbols denote the instructed eye and hand movements for each task. Dark grey circle denotes the peripheral target, presented randomly in one of four locations. White square denotes the cursor feedback provided during each condition. B) Trial timing. Open circles denote non- illuminated target locations. Disappearance of the home target (which occurred at the same time as presentation of the peripheral target) served as the "go-signal" to initiate movement. CHT, center hold time, RT, reaction time, MT, movement time, THT, target hold time. while in the non-standard condition the same targets were presented on the vertical screen, however now participants had to direct a cursor to these targets by sliding their finger along the horizontal tablet (i.e., in a different spatial plane), as well as in the opposite direction of the targets (i.e., feedback reversal). To ensure task comprehension, each participant was given two practice trials per target prior to each condition; they were then asked to perform the experimental task (i.e. five trials to each target) as quickly and accurately as possible. To ensure dissociation between eye and hand movements in the non-standard task, participants were instructed to always look toward the visual target and not at their hand. To reinforce compliance with these task instructions, the eyes were monitored throughout the experiment using a webcam and a reminder was provided in the event that incorrect eye movements were performed.

\section{Imaging data acquisition}

MRI data were acquired using a 3 Tesla Siemens Trio scanner at York University. Sequences included a high-resolution T1-weighted anatomical scan using magnetization prepared rapid gradient echo (MPRAGE) and a whole-brain diffusion-weighted scan with 64 encoding directions. The MPRAGE sequence consisted of 192 sagittal slices with a slice thickness of $1 \mathrm{~mm}$ with no gap, field of view (FOV) of $256 \times 256 \mathrm{~mm}$, and a matrix size of $240 \times 256$, resulting in a voxel resolution of $1 \times 1 \times 1 \mathrm{~mm}^{3}$ [repetition time $(\mathrm{TR})=2300 \mathrm{~ms}$, echo time $(\mathrm{TE})=2.96 \mathrm{~ms}$, flip angle $=9^{\circ}$ ]. For the DTI sequence, 56 axial slices were acquired using diffusion weighted spin-echo single-shot echo planar imaging with a b-value of $1,000 \mathrm{~s} / \mathrm{mm}^{2}$ (including one volume with no diffusion gradient, $b=0 \mathrm{~s} / \mathrm{mm}^{2}$ ), FOV of $192 \times 192 \mathrm{~mm}$, matrix size of $128 \times 128$, and slice thickness of $2 \mathrm{~mm}$ with no gap, resulting in a voxel resolution of $1.5 \times 1.5 \times 2$ $\mathrm{mm}^{3}(\mathrm{TR}=6900 \mathrm{~ms}, \mathrm{TE}=86 \mathrm{~ms})$.

\section{ApoE genotyping}

All older adult participants provided saliva samples for ApoE genotyping conducted at Viaguard Accumetrics (Toronto ON). DNA samples were extracted from the filter paper blots of saliva and standard polymerase chain reaction (PCR) techniques were applied. The PCR products were then analyzed by electrophoresis and visualized under ultraviolet illumination to determine the presence of specific ApoE haplotypes (i.e., E2, E3, and/or E4). 


\section{Kinematic data analysis}

The touchscreen data processing and kinematic outcome measures for the visuomotor tasks used in the current study are described in detail in Hawkins and Sergio [24]. Briefly, movement accuracy was determined by calculating the absolute on-axis (distance) and off-axis (direction) constant errors (CE), which involved computing the average distance between the center of the target and the endpoints of each ballistic movement. Movement precision, or consistency (i.e., variable error; VE), was determined by calculating the standard deviation of the ballistic movement endpoints. The extent to which corrective movements were required in order to reach the target was quantified as the difference between the total path length and the ballistic path length, resulting in a measure of corrective path length (CPL). The time between disappearance of the home target (i.e., the movement 'go signal') and movement onset served as the measure of reaction time (RT), and the time between movement onset and the final movement endpoint upon positioning the cursor inside the peripheral target served as the measure of movement time (MT). In order to minimize the number of correlations between the imaging and behavioral data, kinematic measures from the non-standard task were summarized by calculating z-scores and averaging across the $\mathrm{CE}, \mathrm{VE}$, and $\mathrm{CPL}$ variables to generate a performance error score, and across the RT and MT variables to generate a performance timing score.

\section{Imaging data analysis}

Imaging data were analyzed using the Oxford Centre for Functional Magnetic Resonance Imaging of the Brain (FMRIB) Software Library (FSLhttp://www.fmrib.ox.ac.uk/fsl). In order to test for any hippocampal atrophy across groups, subcortical segmentation was performed on the brain extracted TIweighted MPRAGE data using the FSL tool FIRST [39] and the FSLUTILS program fslstats was used to determine hippocampal volumes.

DTI data were pre-processed using FMRIB's Diffusion Toolbox (FDT). Eddy current and head motion corrections were applied using the affine image registration tool (FLIRT; [40]) and removal of non-brain structures was applied using the brain extraction tool [41]. Maps for fractional anisotropy (FA), mean diffusivity (MD), axial diffusivity (DA), and radial diffusivity (DR) were then extracted using DTIFIT to fit a tensor model to the raw diffusion data and analyzed using whole-brain tract-based spatial statistics
(TBSS; [42]). Specifically, FA data for all subjects used in a particular comparison were aligned to a common space using FMRIB's nonlinear image registration tool (FNIRT), then a mean FA image was created and thinned to generate a mean FA skeleton representing the centers of all tracts common to the group. Each subject's aligned FA data was then projected onto the mean skeleton. The nonlinear warps and projection vectors estimated from the FA images were also applied to MD, DR, and DA using the script provided in TBSS for non-FA images. The resulting data were then fed into voxelwise cross-subject statistics and significant voxels were identified using the Johns Hopkins University (JHU) white matter atlas.

Non-FA diffusion measures were examined in the present study in attempt to understand the underlying alterations driving any observed differences in FA. Specifically, decreased FA with increased MD suggests microstructural declines associated with increased brain water content and macrostructural tissue loss. Whereas, decreased FA without increased MD suggests microstructural changes without gross tissue loss [43]. Reduction in FA accompanied by increased DR may signify loss of myelin integrity, based on experimentally induced myelin degradation in mouse models [44-46]. And lastly, decreased FA and DA, without increased DR, may reflect axonal damage (e.g., wallerian degeneration), which has been demonstrated in both rodent and human experiments [46, 47].

\section{Statistical analysis}

Using SPSS statistical software, a mixed-design analysis of variance (ANOVA) was carried out to compare kinematic measures across the two task conditions (standard/non-standard; repeated), and between the three experimental groups (young/low AD risk/high AD risk). One-way ANOVA tests were also used to compare non-standard error and timing scores, and hippocampal volumes between the three experimental groups. When there were significant effects, post hoc analyses were adjusted for multiple comparisons using Bonferroni correction. ANOVA results were considered statistically significant at $p<0.05$.

FSL's Randomise tool was used to run voxelwise statistics on the TBSS data, with threshold-free cluster enhancement (TFCE) applied to correct for multiple comparisons. Between-group contrasts in both directions for each of the diffusivity measures were tested for young versus low AD risk, young versus high $\mathrm{AD}$ risk, and low $\mathrm{AD}$ risk versus high $\mathrm{AD}$ risk groups. Due to the widespread decline in FA between the 
young and older adult groups, significant FA differences between groups are reported and displayed at $p<0.01$, while significant DR and DA differences are reported and displayed at $p<0.05$ (no significant differences in MD were observed). FSL's cluster tool was used on the significant Randomise results to form clusters and report their sizes, locations in MNI (Montreal Neurological Institute) coordinates, anatomical labels (using the JHU WM atlas), and significance levels. Lastly, mean diffusivity measures were calculated within the clusters that showed significant age-related WM impairment in the high AD risk group. One-way ANOVA tests were used to compare these mean diffusivity measures (i.e., FA/DR/DA) between groups for each WM cluster and post hoc analyses were adjusted for multiple comparisons using Bonferroni correction (alpha-level: $p<0.05$ ). In order to examine the relationship between WM integrity and cognitive-motor performance, mean FA, DR, and DA in these WM clusters were also correlated with error and timing scores from the non-standard task using two-tailed Pearson's $r$ in SPSS. Correlations were considered statistically significant at $p<0.01$.

\section{RESULTS}

\section{Kinematic data}

Consistent with our previous findings [24], significant condition by group interactions driven by

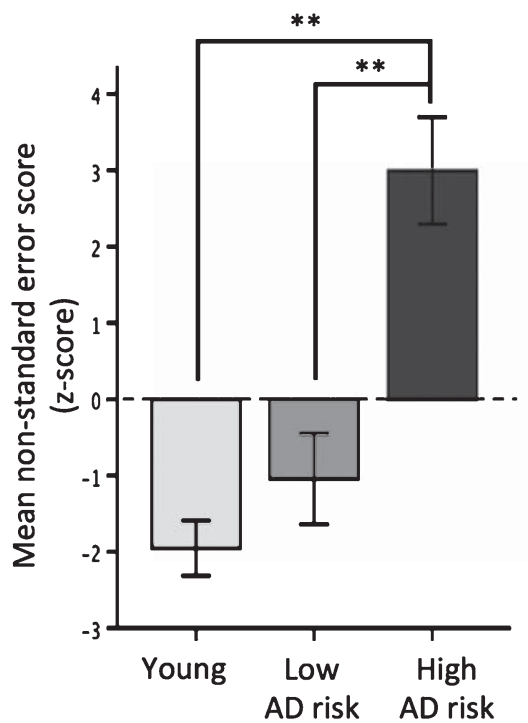

greater performance declines between the standard and non-standard tasks in the high $\mathrm{AD}$ risk group were found for all kinematic measures (On-axis CE: $F_{2,27}=7.36, p=0.003$; Off-axis CE: $F_{2,27}=7.13$, $p=0.003$; VE: $F_{2,27}=11.35, p<0.0001 ; \mathrm{CPL}: F_{2,27}$ $=25.51, p<0.0001$; RT: $F_{2,27}=4.52, p=0.02$; MT: $\left.F_{2,27}=4.97, p=0.015\right)$. Specifically, all three groups performed similarly on the standard visuomotor task, however in the non-standard task on-axis CE, offaxis $\mathrm{CE}, \mathrm{VE}$, and CPL were significantly larger in the high $\mathrm{AD}$ risk group relative to both the low $\mathrm{AD}$ risk and young groups, while RT and MT in the nonstandard task were significantly longer in the high $\mathrm{AD}$ risk group relative to the young group only (see Table 2 for group means and effect sizes). Average zscores calculated to summarize the above error (i.e., $\mathrm{CE}, \mathrm{VE}, \mathrm{CPL}$ ) and timing (i.e., RT and MT) results are displayed in Fig. 2. Accordingly, error z-scores were significantly larger in the high AD risk group relative to both the young and low AD risk groups $\left(F_{2,27}=21.39, p<0.00001 ;\right.$ post-hoc : high AD risk young $=4.95, p<0.00001$, high $\mathrm{AD}$ risk - low $\mathrm{AD}$ risk $=4.04, p<0.0001$ ), and timing $\mathrm{z}$-scores were significantly larger in the high $\mathrm{AD}$ risk group relative to the young group only $\left(F_{2,27}=5.31, p=0.011\right.$; post hoc: high AD risk - young $=1.87, p=0.009$ ).

\section{Imaging data}

The whole-brain TBSS analysis revealed regions of significantly lower WM integrity in both low AD

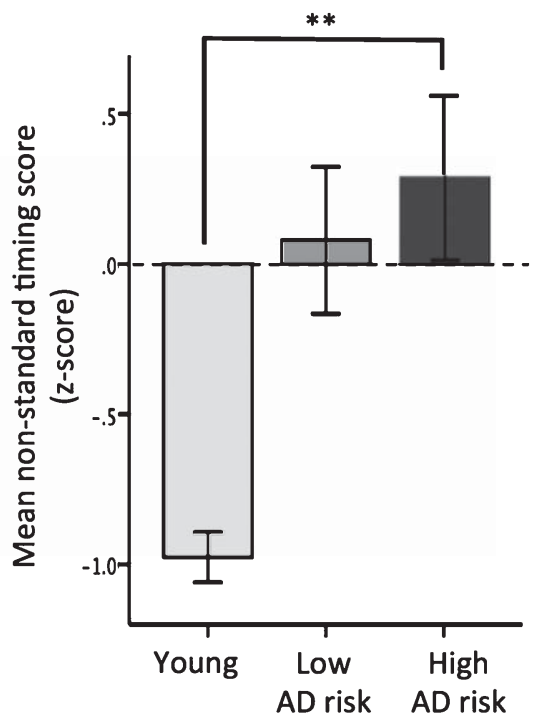

Fig. 2. Mean z-scores for error and timing kinematic measures in the non-standard task across groups (young: light grey bars, low AD risk: medium grey bars, high AD risk: dark grey bars). Note that higher error scores reflect less accuracy and precision and higher timing scores reflect longer reaction and movement times. Means \pm 1 SEM, ${ }^{* *} \leq 0.01$. 


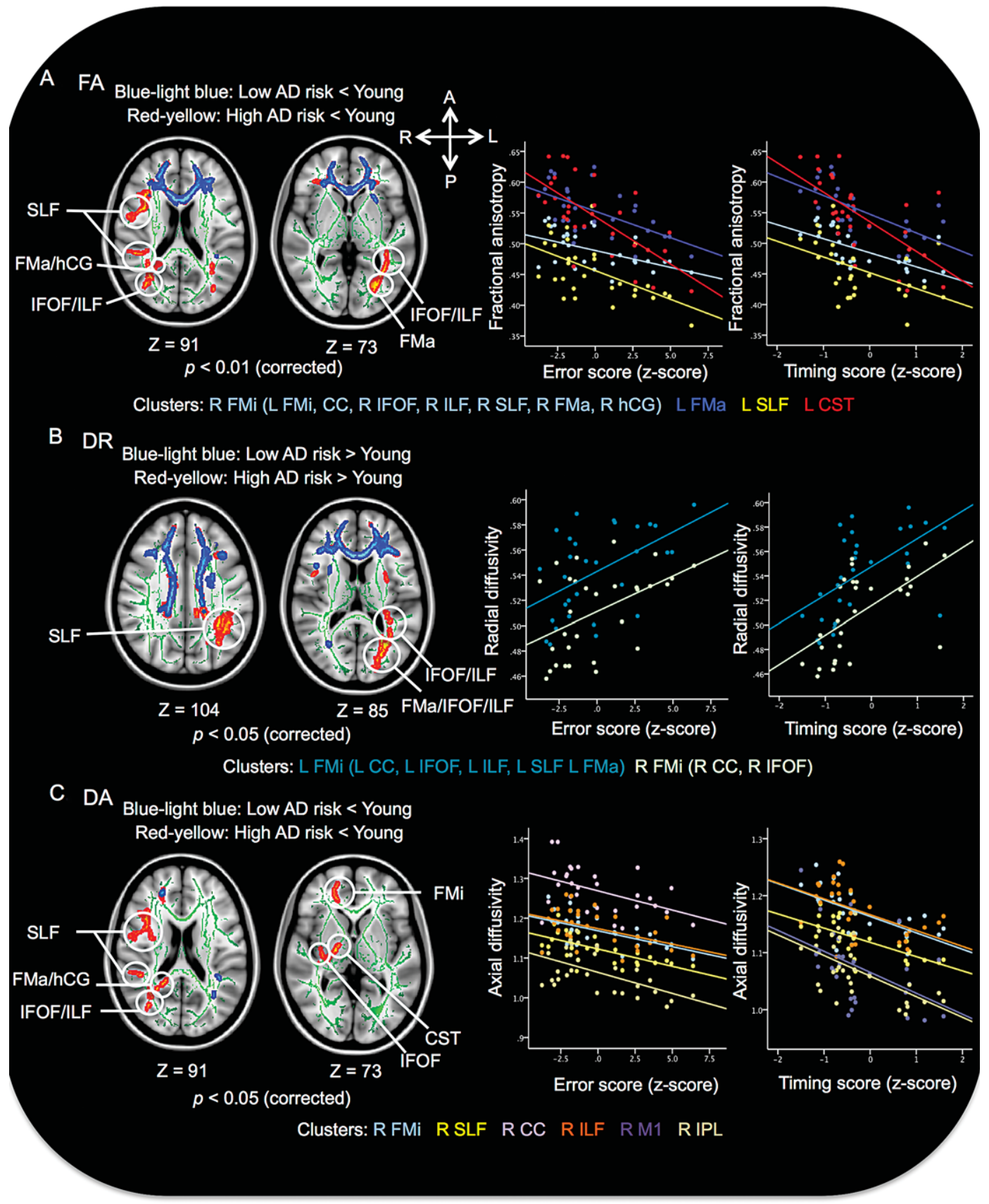

Fig. 3. Left panel: Significant voxelwise between group tract-based spatial statistics (TBSS) results demonstrating (A) lower fractional anisotropy (FA), (B) higher radial diffusivity (DR), and (C) lower axial diffusivity (DA) in both low AD risk (blue-light blue) and high AD risk (red-yellow) older adults relative to young adults. Thickened thresholded $p$-values (tbss_fill) are overlaid on the group mean FA skeleton and displayed on a standard MNI152 brain. Tracts are labeled using the JHU white matter atlas. Right panel: Scatterplots of significant correlations between diffusivity measures [(A) FA, (B) DR, (C) DA] and cognitive-motor performance scores. Correlation statistics are listed in Table 3. R, right; L, left; FMi, forceps minor; CC, corpus callosum; IFOF, inferior fronto-occipital fasciculus; ILF, inferior longitudinal fasciculus; SLF, superior longitudinal fasciculus; FMa, forceps major; hCG, cingulum (hippocampal region); CST, corticospinal tract; M1, primary motor cortex; IPL, inferior parietal lobule.

risk and high $\mathrm{AD}$ risk older adults relative to young adults. These results are displayed in Fig. 3 using FSL's tbss_fill to thicken the thresholded stats results for bet- ter visualization. No significant differences on any of the diffusivity measures were found in the opposite direction (i.e., there were no regions of lower WM 
Table 2

Group means and effect sizes for kinematic measures in each task condition

\begin{tabular}{llccrr}
\hline & \multicolumn{3}{c}{ Group means (SE) } \\
\cline { 2 - 6 } Kinematic measures & Condition & Young & Low AD risk & High AD risk & $\eta p^{2}$ \\
\hline On-axis constant error & Standard & $4.01(0.4)$ & $4.47(0.3)$ & $3.91(0.2)$ & 0.059 \\
& Non-standard & $8.50(1.0)^{\mathrm{a}}$ & $9.76(1.8)^{\mathrm{a}}$ & $18.67(3.1)^{\mathrm{b}}$ & 0.334 \\
Off-axis constant error & Standard & $1.64(0.2)$ & $1.50(0.3)$ & $1.82(0.2)$ & 0.039 \\
& Non-standard & $2.96(0.5)^{\mathrm{a}}$ & $2.96(0.5)^{\mathrm{a}}$ & $5.93(0.6)^{\mathrm{b}}$ & 0.42 \\
Variable error & Standard & $3.47(0.2)$ & $3.01(0.3)$ & $3.28(0.2)$ & 0.067 \\
& Non-standard & $6.41(0.7)^{\mathrm{a}}$ & $9.56(1.2)^{\mathrm{a}}$ & $14.44(1.7)^{\mathrm{b}}$ & 0.440 \\
Corrective path length & Standard & $3.40(0.4)$ & $3.33(0.2)$ & $3.11(0.2)$ & 0.026 \\
& Non-standard & $8.73(1.3)^{\mathrm{a}}$ & $10.45(1.9)^{\mathrm{a}}$ & $32.91(4.0)^{\mathrm{b}}$ & 0.655 \\
Reaction time & Standard & $444(14)^{\mathrm{a}}$ & $508(20)^{\mathrm{b}}$ & $470(17)$ & 0.212 \\
& Non-standard & $603(23)^{\mathrm{a}}$ & $875(62)$ & $1013(142)^{\mathrm{b}}$ & 0.282 \\
Movement time & Standard & $556(28)$ & $661(35)$ & $586(35)$ & 0.168 \\
& Non-standard & $1096(73)^{\mathrm{a}}$ & $1577(169)$ & $2443(496)^{\mathrm{b}}$ & 0.270 \\
\hline
\end{tabular}

Superscripts denote significant differences between group means $(p<0.05)$. Partial eta-squared $\left(\eta \mathrm{p}^{2}\right)$ effect sizes reflect the effect of group within each condition and are based on the linearly independent pairwise comparisons among the estimated marginal means. SE, standard error.

Table 3

Correlations between diffusivity measures and cognitive-motor kinematics

\begin{tabular}{|c|c|c|c|c|c|c|}
\hline & \multicolumn{3}{|c|}{ Error score } & \multicolumn{3}{|c|}{ Timing score } \\
\hline & $r$ & $r^{2}$ & $p$ (2-tailed) & $r$ & $r^{2}$ & $p$ (2-tailed) \\
\hline \multicolumn{7}{|l|}{ Mean FA TBSS clusters (cluster extension) } \\
\hline $\begin{array}{l}\text { R FMi } \\
\text { (L FMi, R/L CC body, R/L SLF, R/L CST) }\end{array}$ & -0.51 & 0.26 & 0.004 & -0.64 & 0.41 & 0.0002 \\
\hline L FMa & -0.59 & 0.35 & 0.001 & -0.64 & 0.41 & 0.0002 \\
\hline L SLF & -0.56 & 0.32 & 0.001 & -0.47 & 0.22 & 0.01 \\
\hline L CST & -0.70 & 0.48 & 0.00002 & -0.66 & 0.44 & 0.0001 \\
\hline Mean DR TBSS clusters (cluster extension) & & & & & & \\
\hline $\begin{array}{l}\text { L FMi } \\
\text { (L CC body, L IFOF, L ILF, L SLF, L FMa) }\end{array}$ & 0.51 & 0.26 & 0.004 & 0.57 & 0.32 & 0.001 \\
\hline $\begin{array}{l}\text { R FMi } \\
\text { (R CC body, R IFOF) }\end{array}$ & 0.47 & 0.22 & 0.009 & 0.61 & 0.37 & 0.001 \\
\hline Mean DA TBSS clusters & & & & & & \\
\hline R FMi & -0.55 & 0.3 & 0.002 & -0.60 & 0.37 & 0.001 \\
\hline R SLF & -0.67 & 0.45 & 0.00005 & -0.59 & 0.34 & 0.001 \\
\hline $\mathrm{R} \mathrm{CC}$ & -0.47 & 0.22 & 0.009 & - & - & - \\
\hline R ILF & -0.47 & 0.22 & 0.009 & -0.50 & 0.25 & 0.006 \\
\hline R IPL & -0.57 & 0.32 & 0.001 & -0.61 & 0.37 & 0.0004 \\
\hline R M1 & - & - & - & -0.49 & 0.24 & 0.008 \\
\hline
\end{tabular}

$\mathrm{R}$, right; L, left; FMi, forceps minor; CC, corpus callosum; SLF, superior longitudinal fasciculus; CST, corticospinal tract; IFOF, inferior fronto-occipital fasciculus; ILF, inferior longitudinal fasciculus; FMa, forceps major; hCG, cingulum (hippocampal region); IPL, inferior parietal lobule; M1, primary motor cortex.

integrity in young relative to older adults). Significantly lower FA (Fig. 3A, left panel; Supplementary Table 1), higher DR (Fig. 3B, left panel; Supplementary Table 2), and lower DA (Fig. 3C, left panel; Supplementary Table 3) in the low AD risk group (blue-light blue) were found primarily in anterior regions including the forceps minor (FMi), body of the CC, and corticospinal tract (CST), while in the high $\mathrm{AD}$ risk group (red-yellow) these age-related declines in WM integrity were more widespread, extending into posterior regions including the forceps major (FMa), IFOF, ILF, SLF, and hippocampal CG (hCG). While the observed age-related declines in WM integrity were more extensive in the high $\mathrm{AD}$ risk group relative to the low AD risk group, this whole-brain analysis did not reveal any significant differences between low and high AD risk groups on any of the diffusivity measures. There were also no significant differences in MD and hippocampal volume between groups.

In support of our hypothesis, a number of significant correlations were found between mean diffusivity measures in isolated WM clusters and cognitive-motor performance scores. Specifically, larger error and timing scores were significantly correlated with lower mean FA in the right FMi (extending into the IFOF, ILF, SLF, FMa, and hCG), left FMa, left SLF, and 

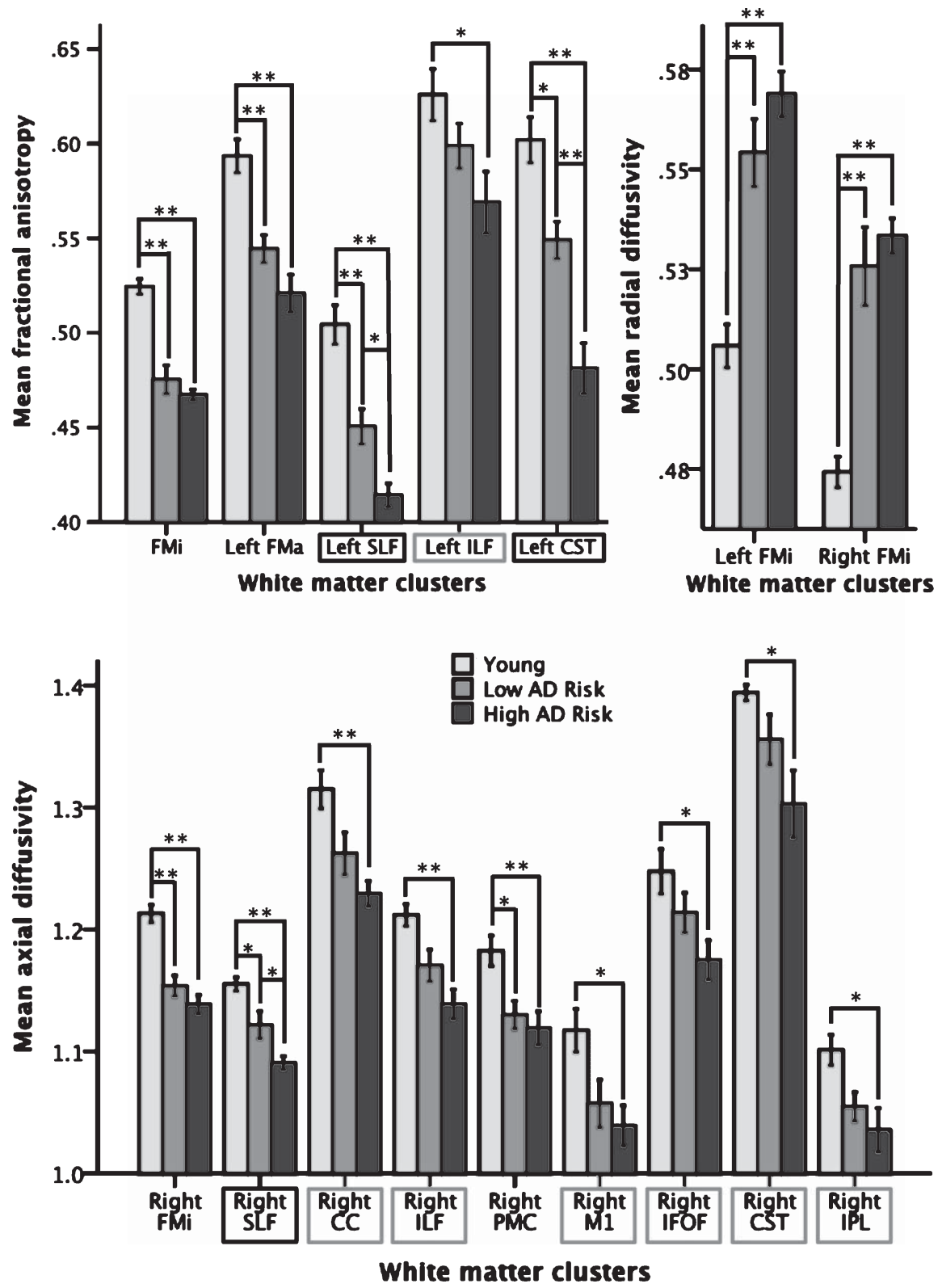

Fig. 4. Mean fractional anisotropy, radial diffusivity, and axial diffusivity measures in isolated white matter (WM) clusters across groups (young: light grey bars, low AD risk: medium grey bars, high AD risk: dark grey bars). Asterisks denote Bonferroni corrected post-hoc analysis results. Means \pm 1 SEM, ${ }^{*} \leq 0.05,{ }^{* *} \leq 0.01$. Dark boxes indicate clusters showing significantly lower WM integrity in high relative to low AD risk participants, and light boxes indicate clusters showing significant age-related WM declines in the high AD risk group only. FMi, forceps minor; FMa, forceps major; SLF, superior longitudinal fasciculus; ILF, inferior longitudinal fasciculus; CST, corticospinal tract; CC, corpus callosum; PMC, premotor cortex; M1, primary motor cortex; IFOF, inferior fronto-occipital fasciculus; IPL, inferior parietal lobule.

left CST (Fig. 3A, right panel). Similarly, we also found significant correlations between larger error and timing scores and higher mean DR in the left FMi (extending into the CC, IFOF, ILF, SLF, and FMa) and right FMi (extending into the $\mathrm{CC}$ and IFOF; Fig. 3B, right panel). Finally, significant correlations were also observed between larger error scores and lower mean DA in the right FMi, SLF, ILF, primary motor cortex (M1), and inferior parietal lobule, and between larger timing scores and lower mean DA in the right FMi, SLF, CC, ILF, and inferior parietal lobule (Fig. 3C, right panel). The statistics for these correlations are listed in Table 3 . 
Importantly, and in accordance with the whole-brain TBSS analysis, the one-way ANOVA tests comparing mean FA, DR, and DA in these isolated WM clusters revealed a stepwise decline in WM integrity across groups (Fig. 4). Specifically, post-hoc analyses revealed significant age-related declines in WM integrity across all clusters and diffusivity measures, which tended to be greater in the high AD risk group. In particular, decreases in FA in the right and left ILF, and decreases in DA in the right CC, M1, IFOF, CST, and inferior parietal lobule were only significant between the young and high AD risk groups and not between the young and low AD risk groups (highlighted by light boxes in Fig. 4). Furthermore, these post-hoc analyses revealed significant differences between the low and high $\mathrm{AD}$ risk groups that the whole-brain analysis was not sensitive enough to detect. Specifically, FA was significantly lower in the left SLF and CST clusters and DA was significantly lower in the right SLF cluster in high relative to low AD risk participants (highlighted by dark boxes in Fig. 4).

\section{DISCUSSION}

The present study revealed declines in WM integrity associated with aging that were more pronounced in older adults at increased AD risk. Specifically, declines in $\mathrm{WM}$ integrity in the low $\mathrm{AD}$ risk group mainly involved the FMi, body of the CC, and CST, while declines in the high AD risk group extended into the FMa, IFOF, ILF, SLF, and hippocampal CG. These findings are consistent with the observation that WM changes in early $\mathrm{AD}$ and mild cognitive impairment typically occur in more posterior regions, whereas changes associated with normal aging tend to occur in anterior regions $[48,49]$. Furthermore, the observation that hippocampal volumes did not differ between groups and MD was not increased in regions with decreased FA is consistent with previous evidence suggesting that alterations in WM integrity in individuals at increase AD risk are not secondary to grey matter atrophy, but rather are the result of microstructural changes without macrostructural tissue loss $[6,7]$.

Our most notable finding, in support of our hypothesis, was the significant relationship between performance on a cognitively demanding visuomotor task and the underlying WM integrity of the brain. Consistent with our previous work [24], we found that measures of timing (reaction and movement time), consistency (variable error), and accuracy (constant error and corrective path length) were significantly impaired in older adults at increased AD risk. While in accordance with changes in sensorimotor control typically observed in normal aging [50-52], older adults at low AD risk only showed slightly increased reaction times, movement times, and variable errors relative to young adults. Correlations between visuomotor performance scores and measures of FA, DR, and DA in several WM clusters (see Table 3) revealed that lower WM integrity was associated with psychomotor slowing, as well as decreased movement accuracy. In combination with the more pronounced declines in WM integrity observed in high AD risk participants in both the whole-brain and isolated WM cluster analyses, these results provide novel evidence for an association between impaired cognitive-motor performance observed in high AD risk participants and underlying WM compromise. While no known previous studies have examined the relationship between WM integrity and cognitive-motor performance in $\mathrm{AD}$, these results are consistent with the finding that lower FA values correspond with worse performance on neuropsychological assessments [53, 54].

In agreement with previous DTI studies [6, 7, 55], the observed WM alterations in participants at increased $\mathrm{AD}$ risk without any clinical symptoms of dementia suggest that disruption to the integrity of WM tracts takes place at an early stage of disease progression. Similar to our results, previous studies in preclinical populations have found declines in FA in the posterior $\mathrm{CC}$, IFOF, and left hippocampus in ApoE4 carriers relative to non-carriers [55], as well as microstructural changes in WM tracts with direct and secondary connections to the medial temporal lobe (i.e., the fornix, CG, ILF, and posterior portions of the IFOF) in cognitively normal women at increased $\mathrm{AD}$ risk due to family history and carrying one or more ApoE4 allele(s) [6, 7]. Earlier studies have also demonstrated reduced glucose metabolism in parietal and temporal areas of ApoE4 carriers over the age of 50 with AD affected relatives [56-58]. Furthermore, individuals with a maternal family history of AD have been shown to exhibit reduced cerebral metabolic rate of glucose (CMRglu) in the posterior cingulate cortex/precuneus, parieto-temporal cortex, frontal cortex, and medial temporal lobe [33]. Taken together, the above results suggest that disconnection between the medial temporal lobe and neocortex, as well as between parietal and frontal regions, may occur very early in the course of AD.

Villain and colleagues [59] have provided direct evidence for this "disconnection hypothesis" using whole-brain voxel-based correlations to assess the 
relationships between hippocampal atrophy, WM integrity, and grey matter metabolism in early AD. Their results revealed that hippocampal atrophy is specifically related to cingulum bundle disruption, which is in turn highly correlated with hypometabolism of the posterior cingulate cortex, middle cingulate gyrus, parahippocampal gyrus, and right temporoparietal association cortex. Other studies using DTI in AD patients have also revealed reduced WM integrity in large-scale networks involving the cingulum bundle [60], as well as fibers connecting prefrontal, medial temporal, and parietal cortices [61]. Based on results such as these, it has been suggested that later myelinating regions with lower oligodendrocyte-to-axon ratios and smaller diameter axons are more vulnerable to myelin degeneration and thus affected earlier in the course of the disease [3, 62, 63]. In accordance with this "retrogenesis model" of AD (i.e., degeneration occurring in a reverse pattern to myelogenesis), Stricker and colleagues [5] have demonstrated lower FA and higher DR values in late-myelinating (ILF, SLF) but not early-myelinating (internal capsule, cerebral peduncles) tracts in $\mathrm{AD}$ patients relative to healthy controls. While direct comparisons between late- and early-myelinating tracts were not made in the present study, the greater age-related declines in WM integrity observed in posterior regions in high AD risk relative to low AD risk participants are consistent with this retrogenesis model. Furthermore, the observation that decreased FA and increased DR occur in similar WM clusters (including those forming the hippocampalparietal and parietal-frontal connections required for visuomotor transformations), and the observation that these changes are correlated with poorer cognitivemotor performance, suggests that myelin degradation may play a role in the visuomotor impairments reported in early AD. However, declines in DA, particularly in the right hemisphere, and significant correlations with performance were also observed, suggesting the additional involvement of axonal disruption.

The present study represents the first attempt to investigate the neural basis of declines in cognitivemotor control observed in older adults at increased $\mathrm{AD}$ risk. In summary, our whole-brain and isolated WM cluster group-level analyses revealed that increased $\mathrm{AD}$ risk was associated with greater and more widespread age-related declines in WM integrity, while our correlational analysis demonstrated significant associations between WM compromise and cognitive-motor performance. It should be noted, however, that the findings presented here only apply to a relatively small sample of women at increased AD risk.
Future work is needed to determine if these results can be generalized to a larger sample including male participants at increased genetic risk for AD. Furthermore, considering the cross-sectional nature of the present study, our findings are not predictive and will require longitudinal follow-up in order to demonstrate whether or not the brain-behavior alterations observed are associated with increased risk of future decline. That being said, not only do these findings in a preclinical population provide support for the view that disruption to WM tracts may be an early identifying feature of $\mathrm{AD}[3,6,7,55,62,63]$, they also provide insight into the impact of AD-related brain alterations on the neural networks underlying complex visuomotor transformations. Importantly, the correlations observed in the present study between kinematic measures on an easily administered visuomotor assessment and microstructural brain alterations suggest that visuomotor performance testing may be applied as a novel behavioral approach to identify individuals at increased AD risk.

\section{ACKNOWLEDGMENTS}

This work was supported by a Canadian Institutes of Health Research operating grant (MOP-74634 to L.E.S.), and a Canadian Institutes of Health Research Banting and Best Canadian Graduate Scholarship, Institute of Aging Special Recognition Award, and Ontario Women's Health Scholars Award to K.M.H.

The authors would like to thank Joy Williams for her technical expertise in the neuroimaging laboratory and Rodrigo Perea for his assistance with the diffusion tensor imaging data analysis.

Authors' disclosures available online (http://www.jalz.com/disclosures/view.php?id=2574).

\section{SUPPLEMENTARY MATERIAL}

The supplementary material is available in the electronic version of this article: http://dx.doi.org/ 10.3233/JAD-142079.

\section{REFERENCES}

[1] Schroeter ML, Stein T, Maslowski N, Neumann J (2009) Neural correlates of Alzheimer's disease and mild cognitive impairment: A systematic and quantitative meta-analysis involving 1351 patients. Neuroimage 47, 1196-1206.

[2] Bai F, Zhang Z, Watson DR, Yu H, Shi Y, Yuan Y, Qian Y, Jia J (2009) Abnormal integrity of association fiber tracts in amnestic mild cognitive impairment. J Neurol Sci 278, 102-106. 
[3] Bartzokis G, Sultzer D, Lu PH, Nuechterlein KH, Mintz J, Cummings JL (2004) Heterogeneous age-related breakdown of white matter structural integrity: Implications for cortical "disconnection" in aging and Alzheimer's disease. Neurobiol Aging 25, 843-851.

[4] Bosch B, Arenaza-Urquijo EM, Rami L, Sala-Llonch R, Junque C, Sole-Padulles C, Pena-Gomez C, Bargallo N, Mollinuevo JL, Bartres-Faz D (2012) Multiple DTI index analysis in normal aging, amnestic MCI and AD. relationship with neuropsychological performance. Neurobiol Aging 33, 61-74.

[5] Stricker NH, Schweinsburg BC, Delano-Wood L, Wierenga CE, Bangen KJ, Haaland KY, Frank LR, Salmon DP, Bondi MW (2009) Decreased white matter integrity in latemyelinating fiber pathways in Alzheimer's disease supports retrogenesis. Neuroimage 45, 10-16.

[6] Gold BT, Powell DK, Andersen AH, Smith CD (2010) Alterations in multiple measures of white matter integrity in normal women at high risk for Alzheimer's disease. Neuroimage 52, 1487-1494.

[7] Smith CD, Chebrolu H, Andersen AH, Powell DA, Lovell MA, Xiong S, Gold BT (2010) White matter diffusion alterations in normal women at risk of Alzheimer's disease. Neurobiol Aging 31, 1122-1131.

[8] Karow DS, McEvoy LK, Fennema-Notestine C, Hagler DJ Jr, Jennings RG, Brewer JB, Hoh CK, Dale AM, Alzheimer's Disease Neuroimaging Initiative (2010) Relative capability of MR imaging and FDG PET to depict changes associated with prodromal and early Alzheimer disease. Radiology 256 , 932-942.

[9] Ewers M, Sperling RA, Klunk WE, Weiner MW, Hampel H (2011) Neuroimaging markers for the prediction and early diagnosis of Alzheimer's disease dementia. Trends Neurosci 34, 430-442.

[10] Battaglia-Mayer A, Ferraina S, Mitsuda T, Marconi B, Genovesio A, Onorati P, Lacquaniti F, Caminiti R (2000) Early coding of reaching in the parietooccipital cortex. $J$ Neurophysiol 83, 2374-2391.

[11] Galati G, Committeri G, Pitzalis S, Pelle G, Patria F, Fattori P, Galletti C (2011) Intentional signals during saccadic and reaching delays in the human posterior parietal cortex. Eur $J$ Neurosci 34, 1871-1885.

[12] Hawkins KM, Sayegh P, Yan X, Crawford JD, Sergio LE (2013) Neural activity in superior parietal cortex during rulebased visual-motor transformations. J Cogn Neurosci 25, 436454.

[13] Sayegh PF, Hawkins KM, Neagu B, Crawford JD, Hoffman KL, Sergio LE (2014) Decoupling the actions of the eyes from the hand alters beta and gamma synchrony within SPL. J Neurophysiol 111, 2210-2221.

[14] Clower DM, West RA, Lynch JC, Strick PL (2001) The inferior parietal lobule is the target of output from the superior colliculus, hippocampus, and cerebellum. J Neurosci $\mathbf{2 1}$, 6283-6291.

[15] Crawford JD, Henriques DY, Medendorp WP (2011) Threedimensional transformations for goal-directed action. Annu Rev Neurosci 34, 309-331.

[16] Johnson PB, Ferraina S, Bianchi L, Caminiti R (1996) Cortical networks for visual reaching: Physiological and anatomical organization of frontal and parietal lobe arm regions. Cereb Cortex 6, 102-119.

[17] Wise SP, di Pellegrino G, Boussaoud D (1996) The premotor cortex and nonstandard sensorimotor mapping. Can J Physiol Pharmacol 74, 469-482.
[18] de Boer C, Mattace-Raso F, van der Steen J, Pel JJ (2014) Mini-mental state examination subscores indicate visuomotor deficits in Alzheimer's disease patients: A cross-sectional study in a Dutch population. Geriatr Gerontol Int 14, 880-885.

[19] Ghilardi MF, Alberoni M, Marelli S, Rossi M, Franceschi M, Ghez C, Fazio F (1999) Impaired movement control in Alzheimer's disease. Neurosci Lett 260, 45-48.

[20] Ghilardi MF, Alberoni M, Rossi M, Franceschi M, Mariani C, Fazio F (2000) Visual feedback has differential effects on reaching movements in Parkinson's and Alzheimer's disease. Brain Res 876, 112-123.

[21] Hawkins KM, Sergio LE (2014) Visuomotor impairments in older adults at increased Alzheimer's disease risk. J Alzheimers Dis 42, 607-621.

[22] Salek Y, Anderson ND, Sergio L (2011) Mild cognitive impairment is associated with impaired visual-motor planning when visual stimuli and actions are incongruent. Eur Neurol 66, 283-293.

[23] Tippett WJ, Sergio LE (2006) Visuomotor integration is impaired in early stage Alzheimer's disease. Brain Res 1102, 92-102.

[24] Tippett WJ, Krajewski A, Sergio LE (2007) Visuomotor integration is compromised in Alzheimer's disease patients reaching for remembered targets. Eur Neurol 58, 1-11.

[25] Tippett WJ, Sergio LE, Black SE (2012) Compromised visually guided motor control in individuals with Alzheimer's disease: Can reliable distinctions be observed? J Clin Neurosci 19, 655-660.

[26] Verheij S, Muilwijk D, Pel JJ, van der Cammen TJ, MattaceRaso FU, van der Steen J (2012) Visuomotor impairment in early-stage Alzheimer's disease: Changes in relative timing of eye and hand movements. J Alzheimers Dis 30, 131-143.

[27] Georgopoulos AP (2000) Neural aspects of cognitive motor control. Curr Opin Neurobiol 10, 238-241.

[28] Alzheimer's Association (2012) Alzheimer's disease facts and figures. Alzheimers Dement 8, 131-168.

[29] Fratiglioni L, Ahlbom A, Viitanen M, Winblad B (1993) Risk factors for late-onset Alzheimer's disease: A populationbased, case-control study. Ann Neurol 33, 258-266.

[30] Green RC, Cupples LA, Go R, Benke KS, Edeki T, Griffith PA, Williams M, Hipps Y, Graff-Radford N, Bachman D, Farrer LA, MIRAGE Study Group (2002) Risk of dementia among white and African American relatives of patients with Alzheimer disease. JAMA 287, 329-336.

[31] Mayeux R, Sano M, Chen J, Tatemichi T, Stern Y (1991) Risk of dementia in first-degree relatives of patients with Alzheimer's disease and related disorders. Arch Neurol 48, 269-273.

[32] Lautenschlager NT, Cupples LA, Rao VS, Auerbach SA, Becker R, Burke J, Chui H, Duara R, Foley EJ, Glatt SL, Green RC, Jones R, Karlinsky H, Kukull WA, Kurz A, Larson EB, Martelli K, Sadovnick AD, Volicer L, Waring SC, Growdon JH, Farrer LA (1996) Risk of dementia among relatives of Alzheimer's disease patients in the MIRAGE study: What is in store for the oldest old? Neurology 46, 641-650.

[33] Honea RA, Swerdlow RH, Vidoni ED, Burns JM (2011) Progressive regional atrophy in normal adults with a maternal history of Alzheimer disease. Neurology 76, 822-829.

[34] Mosconi L, Brys M, Switalski R, Mistur R, Glodzik L, Pirraglia E, Tsui W, De Santi S, de Leon MJ (2007) Maternal family history of Alzheimer's disease predisposes to reduced brain glucose metabolism. Proc Natl Acad Sci U S A 104, 19067-19072.

[35] Mosconi L, Glodzik L, Mistur R, McHugh P, Rich KE, Javier E, Williams S, Pirraglia E, De Santi S, Mehta PD, Zinkowski 
R, Blennow K, Pratico D, de Leon MJ (2010) Oxidative stress and amyloid-beta pathology in normal individuals with a maternal history of Alzheimer's. Biol Psychiatry 68, 913-921.

[36] Carter CL, Resnick EM, Mallampalli M, Kalbarczyk A (2012) Sex and gender differences in Alzheimer's disease: Recommendations for future research. J Womens Health 21, 1018-1023.

[37] Schmidt R, Kienbacher E, Benke T, Dal-Bianco P, Delazer M, Ladurner G, Jellinger K, Marksteiner J, Ransmayr G, Schmidt H, Stögmann E, Friedrich J, Wehringer C (2008) Sex differences in Alzheimer's disease. Neuropsychiatr 22, $1-15$.

[38] Reilly SL, Ferrell RE, Sing CF (1994) The gender-specific apolipoprotein $\mathrm{E}$ genotype influence on the distribution of plasma lipids and apolipoproteins in the population of rochester, MN. III. correlations and covariances. Am J Hum Genet 55, 1001-1018.

[39] Patenaude B, Smith SM, Kennedy DN, Jenkinson M (2011) A bayesian model of shape and appearance for subcortical brain segmentation. Neuroimage 56, 907-922.

[40] Jenkinson M, Bannister P, Brady M, Smith S (2002) Improved optimization for the robust and accurate linear registration and motion correction of brain images. Neuroimage 17, 825-841.

[41] Smith SM (2002) Fast robust automated brain extraction. Hum Brain Mapp 17, 143-155.

[42] Smith SM, Jenkinson M, Johansen-Berg H, Rueckert D, Nichols TE, Mackay CE, Watkins KE, Ciccarelli O, Cader MZ, Matthews PM, Behrens TE (2006) Tract-based spatial statistics: Voxelwise analysis of multi-subject diffusion data. Neuroimage 31, 1487-1505.

[43] Sen PN, Basser PJ (2005) A model for diffusion in white matter in the brain. Biophys $J \mathbf{8 9}, 2927-2938$.

[44] Song SK, Sun SW, Ju WK, Lin SJ, Cross AH, Neufeld AH (2003) Diffusion tensor imaging detects and differentiates axon and myelin degeneration in mouse optic nerve after retinal ischemia. Neuroimage 20, 1714-1722.

[45] Song SK, Yoshino J, Le TQ, Lin SJ, Sun SW, Cross AH, Armstrong RC (2005) Demyelination increases radial diffusivity in corpus callosum of mouse brain. Neuroimage 26, 132-140.

[46] Sun SW, Liang HF, Trinkaus K, Cross AH, Armstrong RC, Song SK (2006) Noninvasive detection of cuprizone induced axonal damage and demyelination in the mouse corpus callosum. Magn Reson Med 55, 302-308.

[47] Concha L, Gross DW, Wheatley BM, Beaulieu C (2006) Diffusion tensor imaging of time-dependent axonal and myelin degradation after corpus callosotomy in epilepsy patients. Neuroimage 32, 1090-1099.

[48] Head D, Buckner RL, Shimony JS, Williams LE, Akbudak E, Conturo TE, McAvoy M, Morris JC, Snyder AZ (2004) Differential vulnerability of anterior white matter in nondemented aging with minimal acceleration in dementia of the Alzheimer type: Evidence from diffusion tensor imaging. Cereb Cortex 14, 410-423.

[49] Head D, Snyder AZ, Girton LE, Morris JC, Buckner RL (2005) Frontal-hippocampal double dissociation between normal aging and Alzheimer's disease. Cereb Cortex 15, 732-739.

[50] Spirduso WW (1975) Reaction and movement time as a function of age and physical activity level. J Gerontol 30, 435-440.
[51] Spirduso WW, Clifford P (1978) Replication of age and physical activity effects on reaction and movement time. J Gerontol 33, 26-30.

[52] Yan JH, Thomas JR, Stelmach GE, Thomas KT (2000) Developmental features of rapid aiming arm movements across the lifespan. J Mot Behav 32, 121-140.

[53] Mielke MM, Kozauer NA, Chan KC, George M, Toroney J, Zerrate M, Bandeen-Roche K, Wang MC, Vanzijl P, Pekar JJ, Mori S, Lyketsos CG, Albert M (2009) Regionally-specific diffusion tensor imaging in mild cognitive impairment and Alzheimer's disease. Neuroimage 46, 47-55.

[54] Rose SE, McMahon KL, Janke AL, O'Dowd B, de Zubicaray G, Strudwick MW, Chalk JB (2006) Diffusion indices on magnetic resonance imaging and neuropsychological performance in amnestic mild cognitive impairment. J Neurol Neurosurg Psychiatry 77, 1122-1128.

[55] Persson J, Lind J, Larsson A, Ingvar M, Cruts M, Van Broeckhoven C, Adolfsson R, Nilsson LG, Nyberg L (2006) Altered brain white matter integrity in healthy carriers of the APOE epsilon4 allele: A risk for AD? Neurology 66, 1029-1033.

[56] Reiman EM, Caselli RJ, Yun LS, Chen K, Bandy D, Minoshima S, Thibodeau SN, Osborne D (1996) Preclinical evidence of Alzheimer's disease in persons homozygous for the epsilon 4 allele for apolipoprotein E. N Engl J Med 334, 752-758.

[57] Small GW, Mazziotta JC, Collins MT, Baxter LR, Phelps ME, Mandelkern MA, Kaplan A, La Rue A, Adamson CF, Chang L (1995) Apolipoprotein E type 4 allele and cerebral glucose metabolism in relatives at risk for familial Alzheimer disease. JAMA 273, 942-947.

[58] Small GW, Ercoli LM, Silverman DH, Huang SC, Komo S, Bookheimer SY, Lavretsky H, Miller K, Siddarth P, Rasgon NL, Mazziotta JC, Saxena S, Wu HM, Mega MS, Cummings JL, Saunders AM, Pericak-Vance MA, Roses AD, Barrio JR, Phelps ME (2000) Cerebral metabolic and cognitive decline in persons at genetic risk for Alzheimer's disease. Proc Natl Acad Sci U S A 97, 6037-6042.

[59] Villain N, Desgranges B, Viader F, de la Sayette V, Mezenge F, Landeau B, Baron JC, Eustache F (2008) Relationships between hippocampal atrophy, white matter disruption, and gray matter hypometabolism in Alzheimer's disease. $J$ Neurosci 28, 6174-6181.

[60] Zhang Y, Schuff N, Jahng GH, Bayne W, Mori S, Schad L, Mueller S, Du AT, Kramer JH, Yaffe K, Chui H, Jagust WJ, Miller BL, Weiner MW (2007) Diffusion tensor imaging of cingulum fibers in mild cognitive impairment and Alzheimer disease. Neurology 68, 13-19.

[61] Sexton CE, Kalu UG, Filippini N, Mackay CE, Ebmeier KP (2011) A meta-analysis of diffusion tensor imaging in mild cognitive impairment and Alzheimer's disease. Neurobiol Aging 32, 2322.e5-18.

[62] Bartzokis G (2004) Age-related myelin breakdown: A developmental model of cognitive decline and Alzheimer's disease. Neurobiol Aging 25, 5-18.

[63] Reisberg B, Franssen EH, Souren LE, Auer SR, Akram I, Kenowsky S (2002) Evidence and mechanisms of retrogenesis in Alzheimer's and other dementias: Management and treatment import. Am J Alzheimers Dis Other Demen 17, 202-212. 